\title{
Article
}

Mycosphere

Doi 10.5943/mycosphere/7/5/9

Copyright $($ ) Guizhou Academy of Agricultural Sciences

\section{Additions to the quadrate-spored Entoloma (Agaricales) in Kerala State, India}

\author{
Pradeep $\mathrm{CK}^{1 *}$, Vrinda $\mathrm{KB}^{1}$, Bijeesh $\mathrm{C}^{\mathbf{1}}$ and Baroni $\mathrm{TJ}^{\mathbf{2}}$ \\ ${ }^{1}$ Jawaharlal Nehru Tropical Botanic Garden \& Research Institute, Palode, Thiruvananthapuram, 695 562, Kerala, \\ India \\ ${ }^{2}$ Department of Biological Sciences, State University of New York, College at Cortland, New York, USA - 13045
}

Pradeep CK, Vrinda KB, Bijeesh C, Baroni TJ 2016 - Additions to the quadrate-spored Entoloma (Agaricales) in Kerala State, India. Mycosphere 7(5), 642-648, Doi 10.5943/mycosphere/7/5/9

\begin{abstract}
Two new quadrate spored species of Entoloma are described from Kerala, India based on morphological characters. Complete morphological descriptions along with photographs of habit and microstructures are provided. The new species are compared and discussed with morphologically similar species.
\end{abstract}

Key words - Agarics - Basidiomycota - entolomatoid fungi - quadrate spores - taxonomy

\section{Introduction}

Entoloma s.l. (Entolomataceae, Agaricales, Basidiomycota) is a large genus represented by about 1282 species world-wide (Kirk et al. 2008). Previous works on the entolomatoid fungi of Kerala resulted in the documentation of about 62 taxa (Manimohan et al. 1995, 2002, 2006, Raj \& Manimohan 2012, Pradeep et al. 2012, 2013, Raj et al. 2014, 2015). Among these 62 taxa, 15 belong to the quadrate spored category, which seems to be predominant in the region. During our continuing studies on the entolomatoid fungi of Kerala State India, we came across two new species of Entoloma with quadrate basidiospores that are formally described here.

\section{Materials \& Methods}

Gross morphological descriptions are based exclusively on fresh materials collected from Kerala State, India. Microscopic characters were studied on dried material using hand cut sections of basidiomata revived in $3 \%$ solution of $\mathrm{KOH}$, stained with $1 \%$ Congo red and examined under a Leica DME1000 compound microscope. The mean quotient (Q) of spore length divided by spore width was calculated from measurements of 20 basidiospores; the hilar appendix was excluded in the measurement of the spore length. Methods used in the examination of microscopic features are those of Largent et al. (1977). Colour notations refer to Kornerup \& Wanscher (1978). Descriptive terms follow Vellinga (1988). The generic and subgeneric classification followed is that of Noordeloos (1981). All holotypes are deposited at Central National Herbarium (CAL), Kolkata, India and the CAL accession numbers are provided. All isotypes and additional materials examined are deposited at the Mycological Herbarium of Jawaharlal Nehru Tropical Botanic Garden and Research Institute, Trivandrum (TBGT). 


\section{Results}

\section{Taxonomy}

Entoloma flavostipitatum C.K. Pradeep \& K.B. Vrinda, sp.nov. Fig.1

MycoBank MB815088 Facesoffungi number: FoF 02623

Diagnosis - Differs from Entoloma brunneum Petch in having smaller omphalioid basidiomata, yellowish subdecurrent lamellae, yellowish smooth stipe, smaller basidiospores and presence of caulocystidia.

Holotype - INDIA, Kerala State, Trivandrum District, Palode, JNTBGRI campus, 18 Jul 2011, Bijeesh, TBGT13699 (CAL1284, Holotype).

Etymology - flavostipitatum - refers to the yellow stipe.

Basidiomata small to medium, omphalioid. Pileus8-30 mm diam., convex becoming planoconvex to applanate with a shallow depression at centre; surface chocolate to teak brown $(6 \mathrm{~F} 4,6 \mathrm{~F} 5)$ at first, becoming paler and grayish-brown or hair brown to clay color $(5 \mathrm{E} 4,5 \mathrm{D} 5,6 \mathrm{E} 3,6 \mathrm{E} 4)$ when mature, smooth to naked eye, finely squamulose (under lens) at the disc, sparsely squamulose towards margin, pellucid striate up to half way, nonhygrophanous, rarely splitting when mature, moist; margin incurved becoming straight, entire to incised. Lamellae broadly adnate to subdecurrent, or with a subdecurrent tooth, pale yellow to cream $(3 \mathrm{~A} 2,3 \mathrm{~A} 3,4 \mathrm{~A} 3)$, becoming pale pink, up to $4 \mathrm{~mm}$ wide, close to sub-crowded with lamellulae of different lengths; edge brownish (in some basidiomata). Stipe $10-35 \times 2-4 \mathrm{~mm}$, central, cylindric to rarely compressed, curved, hollow, brittle, tapering up from a slightly broad base; surface pale yellow to butter yellow (3A3,4A4,4A5), smooth, glabrous; white mycelial mat and mycelial chords present. Context thin, up to $1 \mathrm{~mm}$ thick, white or with a brownish shade, soft. Odor mild, agreeable, not characteristic. Spore print brownish orange (7C5).

Basidiospores 6.5-8.5 × 5.5-7.5 $\mu \mathrm{m}$, avL $=7.22$, avW $=6.5, \mathrm{Q}=1-1.3$, avQ $=1.12$, quadrate in profile, occasionally pentagonal. Basidia 26-33 $\times 9.5-12.5 \mu \mathrm{m}$, clavate, 4-spored. Lamella edge sterile with crowded cystidia. Cystidia tramal in origin, 30-44 $\times 6-8.5 \mu \mathrm{m}$, cylindroclavate, thin-walled with yellowish brown contents. Pleurocystidia absent. Hymenophoral trama regular; hyphae 5-14 $\mu \mathrm{m}$ wide, thin-walled, hyaline. Subhymenium not well developed, thin, hyphal. Pileal trama interwoven, thin-walled; hyphae similar to hymenophoral trama. Pileipellis a cutis of 5-10 $\mu \mathrm{m}$ wide hyphae, becoming a trichoderm towards the center. Trichoderm elements 30-37.6 $\times 10-16 \mu \mathrm{m}$, clavate to cylindroclavate, thin-walled with brown intracellular contents. Stipitipellis a cutis composed of thin-walled hyphae, 5-8.5 $\mu \mathrm{m}$ wide, with pale yellowish contents. Caulocystidia present in tufts on the upper part of the stipe, $21.5-38 \times 5-9.5 \mu \mathrm{m}$, cylindro-clavate, thin-walled with yellowish contents. Oleiferous hyphae present in stipe trama. Clamp connections present in all tissues.

Habit and habitat - Solitary, scattered or in groups on mud wall, on earthworm mounts or on ant hills in evergreen forest, July-November.

Additional materials examined - India, Kerala State, Trivandrum District, Palode, JNTBGRI campus, 19 Jul 2011, Bijeesh TBGT13715; 21 Aug 2012, Bijeesh TBGT14214; 4 Aug 2014, Bijeesh TBGT15269; 4 Nov 2014, Bijeesh TBGT15460.

\section{Discussion}

Entoloma flavostipitatum is characterized by a set of features such as small to medium brown omphalioid basidiomata; brown convex, depressed finely squamulose and nonhygrophanous pileus with a depressed disc; broadly adnate to subdecurrent yellowish lamellae; yellow, smooth stipe; quadrate basidiospores; sterile lamellae edge with cystidiform elements with brown intracellular pigment; pileipellis a cutis with a transition to a trichoderm towards the disc, presence of caulocystidia and presence of clamp connections in all tissues. 

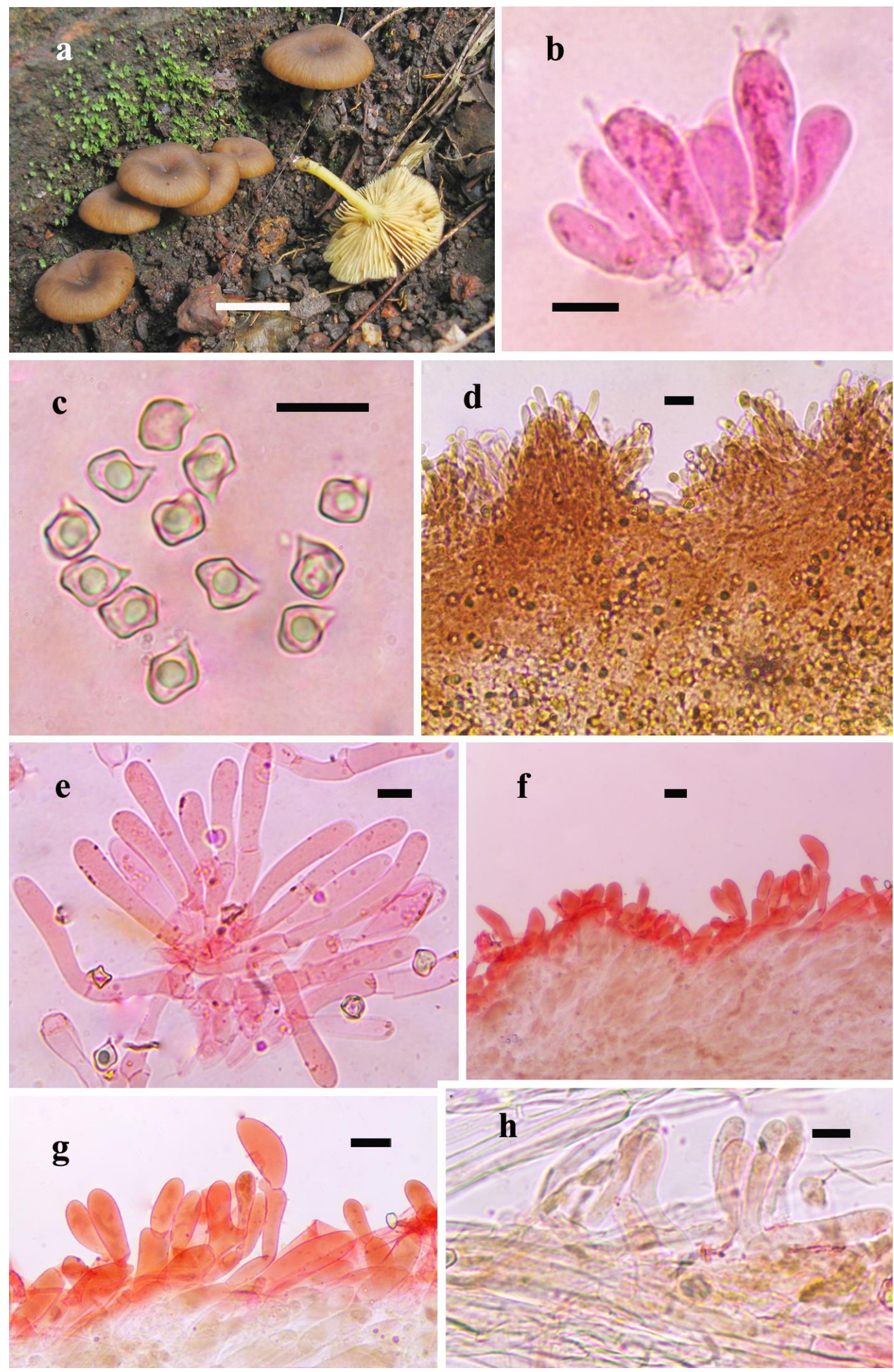

Fig.1 - Entoloma flavostipitatum(CAL1284). a. Basidiomata in situ. b. Basidia. c. Spores. d. Lamella edge. e. Cystidia. f. Pileipellis. g. Trichodermial elements. h. Caulocystidia. Scale bars: $a=$ $10 \mathrm{~mm} ; \mathrm{b}-\mathrm{e}, \mathrm{h}=10 \mu \mathrm{m} ; \mathrm{f}-\mathrm{g}=20 \mu \mathrm{m}$.

Among the quadrate spored species described by different workers (Hesler 1967, Horak 1976, 1977, Pegler 1986, Gates \& Noordeloos 2007, Noordeloos 1992, 2004, Noordeloos \& Hausknecht 2007, Noordeloos \& Morozova 2010) Entoloma brunneum and E. infundibuliforme 
Petch share some common features with the present material. The former has a similar brown squamulose pileus, brownish lamella edges, almost similar sized quadrate basidiospores, cylindro-clavate cheilocystidia with brown contents and presence of clamp connections. Entoloma brunneum however, has much larger basidiomata with a conical, umbonate, squarrose, hygrophanous pileus, adnexed pink lamellae, white to brown squamulose stipe, slightly larger spores, and lack of caulocystidia. Entoloma infundibuliforme though similar in many respects, differs by its larger basidiomata with scurfy squamulose pileus, pink lamellae with concolorous edges, white to brown innately fibrillose stipe, fusoid cheilocystidia with acute apex and lack of caulocystidia.

Some of the recently described brown species with quadrate basidiospores from Kerala includes E. brunneoquadratum Manim. \& Noordel., E. perinfundibuliforme Manim. \& Noordel., E. keralense Manim. \& Noordel. (Manimohan et al. 2006), E. lomavrithum KNA Raj \& Manim. (Raj \& Manimohan 2012), E. brunneopapillatum C.K. Pradeep \& K.B. Vrinda, E. brunneosquamulosum C.K. Pradeep \& K.B. Vrinda, and E. brunneocarnosum C.K.Pradeep \& K.B.Vrinda (Pradeep et al. 2013). Medium sized basidiomata with a smooth glabrous translucently striate pileus, free pink lamellae, brown stipe, fertile lamella edge and absence of clampconnections distinguish E. brunneoquadratum from the present species. Large, fleshy basidiomata with a deeply depressed pileus, adnexed lamellae, white appressed fibrillose stipe, lamella edge with dense strand of hyphae and pileipellis a disrupted cutis separates E. perinfundibuliforme from the present taxon. Entoloma keralense is distinct by its small, delicate, fibrillose squamulose pileus, ovoid to ventricose rostrate pleurocystidia and pileipellis a cutis. Entoloma lomavrithum differs mainly by its small basidiomata with a tomentose pileus and stipe and absence of clamp connections. Entoloma brunneopapillatum is characterized by its small to medium mycenoid basidiomata, papillate, hygrophanous, glabrous pileus, adnate pink lamellae, brown brittle stipe, and fertile lamellae edge. Large tricholomatoid basidiomata, a brown squamulose pileus with subacute umbo brown squamulose stipe, and the production of colorless watery exudates on cutting the flesh of the basidiomata makes E. brunneosquamulosum distinct. Clitocyboid basidiomata with deeply depressed, smooth, glabrous, moist to slimy pellucid-striate pileus, adnate lamellae, slightly gelatinized pileipellis and absence of clamp connections characterize E. brunneocarnosum.

The omphalioid basidiomata with a depressed squamulose brown pileus, marginate lamella edges, heterodiametric basidiospores, pileipellis a cutis passing to a trichoderm and presence of clamp connections indicates that the new species belongs to subgenus Leptonia section Griseorubida (Noordeloos 1981).

Entoloma flavoquadratum C.K.Pradeep \& K.B.Vrinda, sp. nov. Fig. 2 MycoBankMB815089 Facesoffungi number: FoF 02624

Diagnosis -Differs from the similar Entoloma cremeoluteum (Largent) Noordel. \& CoDavid by larger basidiomata, larger basidiospores, cylindro-clavate cheilocystidia and absence of pleurocystidia.

Holotype - INDIA, Kerala State, Kollam District, Thenmala, 22 Jul 2011, CK Pradeep TBGT13739(CAL 1285, Holotype)

Etymology-flavoquadratum - refers to the yellow basidiomata with quadrate basidiospores.

Basidiomata medium to large, tricholomatoid. Pileus 30-63 mm diam., convex with abroad obtuse umbo, become plano-convex to plane with or without a small obtuse umbo; surface pale yellow to light yellow (2A3, 2A4, 4A3, 4A4), non-striate, nonhygrophanous, smooth, glabrous, rarely cracked to expose the underlying yellowish context, slightly greasy to touch when moist; margin straight, entire, exceeding the lamellae, wavy, non-striate. Lamellae adnexed, yellowish white $(2 \mathrm{~A} 2,2 \mathrm{~A} 3,4 \mathrm{~A} 2)$, up to $6 \mathrm{~mm}$ wide, close with lamellulae of 4-5 lengths; edge concolorous, uneven, eroded. Stipe 40-92 × 6-10 mm, central, cylindric, twisted, silky striate, stuffed becoming hollow, tapering up from a slightly broad base; surface concolorous with pileus, smooth, glabrous; white mycelial mat none. Context concolorous to the pileus, up to $3 \mathrm{~mm}$, soft. Odor mild, not characteristic. Spore print orange white (5A2). 

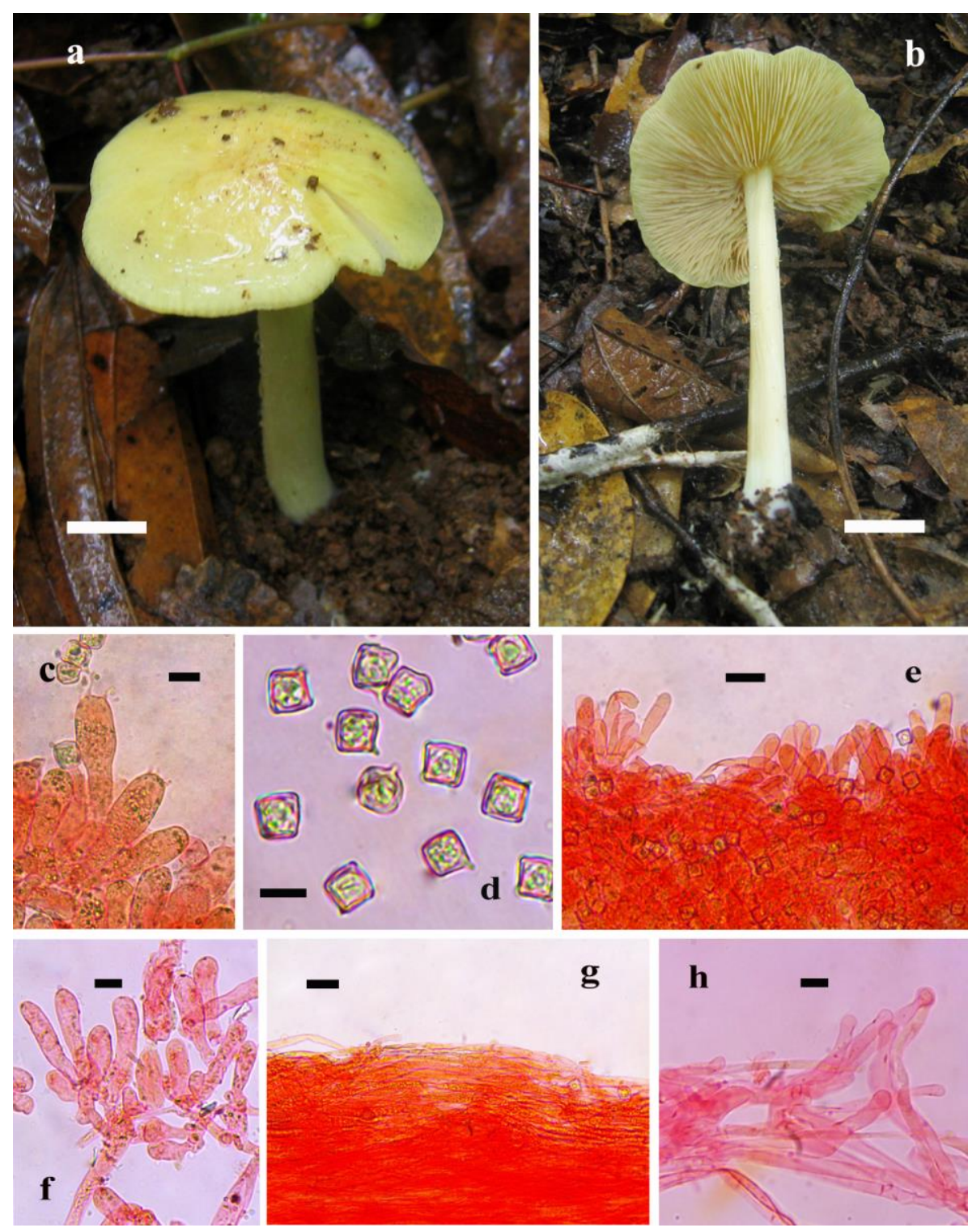

Fig.2 -Entoloma flavoquadratum(CAL1285). a-b. Basidioma in situ c. Basidia. d. Spores. e. Lamella edge. f. Cheilocystidia. g. Pileipellis. h. Caulocystidia.Scale bars:a-b $=10 \mathrm{~mm} ; \mathrm{c}-\mathrm{d}, \mathrm{f}, \mathrm{h}$ $=10 \mu \mathrm{m} ; \mathrm{e}, \mathrm{g}=20 \mu \mathrm{m}$.

Basidiospores 9-11.5 $\times 8-11 \mu \mathrm{m}$, avL $=10.1$, avW $=10.0, \mathrm{Q}=1-1.2$, avQ $=1.04$, cuboid in profile. Basidia 52-54.5 $\times 14.5-17 \mu \mathrm{m}$, clavate, 4-spored with dense shiny granular yellowish contents. Lamella edge sterile with crowded cheilocystidia. Cheilocystidia 18.5-36 × 6.5-11 $\mu \mathrm{m}$, cylindrical to cylindro-clavate, often slightly strangulated, thin-walled, hyaline. Pleurocystidia absent. Hymenophoral trama regular; hyphae 4.5-8 $\mu \mathrm{m}$ wide, thin-walled, hyaline. Subhymenium thin, interwoven. Pileal trama interwoven; hyphae similar to hymenophoral trama. Pileipellis a cutis with slightly gelatinized hyphae, 4-7(-10) $\mu \mathrm{m}$ wide, thin-walled, with dense shiny granular yellowish contents. Stipitipellis a cutis composed of thin-walled parallel hyphae, 3-6.5 $\mu \mathrm{m}$ wide, with pale yellowish contents. Caulocystidia present on the upper part. Caulocystidia 36-57.5 $\times 4$ $6.4 \mu \mathrm{m}$, cylindro-clavate, rarely strangulated, thin-walled with pale yellowish contents. Oleiferous hyphae present. Clamp connections present in all tissues. September.

Habit and habitat - Solitary, scattered on soil among litter in evergreen forest, June -

Additional materials examined - India, Kerala State, Kollam District, Thenmala, 4 Sep 2012, Bijeesh TBGT14245; 19 Jun 2013, Bijeesh TBGT14571. 


\section{Discussion}

Entoloma flavoquadratum is distinctive by its medium to large tricholomatoid, yellowish basidiomata, convex becoming applanate, nonhygrophanous, glabrous greasy pileus, yellowish adnexed lamellae, yellowish, smooth stipe, quadrate basidiospores, sterile lamellae edge, slightly gelatinized cuticular pileipellis, presence of caulocystidia and presence of clamp connections in all tissues.

Entoloma flavoquadratum has much in common with E. cremeoluteum described from California (Largent 1994) with regard to the yellowish pileus and stipe, quadrate basidiospores and presence of clamp connections. Apart from these, E. cremeoluteum is distinct by its smaller basidiomata (pileus up to $27 \mathrm{~mm}$ diam.), broadly campanulate hygrophanous pileus, smaller basidiospores $(7.2-10.4 \times 6.1-9.2 \mu \mathrm{m})$, rostrate-ventricose cheilocystidia and pleurocystidia, absence of caulocystidia and presence of rare clamp connections. Entoloma pallidoflavum (Henn. \& E. Nyman) E. Horak (Horak 1976), is related in some macro and microscopic features but can well be separated by its conical to companulate whitish yellow pileus with appressed fibrillose rimose pileus, sulcate margin, adnexed to adnate lamellae, exudation of a clear latex on cutting of the basidiomata, smaller basidiospores $(7.5-10 \mu \mathrm{m})$, large cheilocystidia $(50-120 \times 5-12 \mu \mathrm{m})$ and lack of caulocystidia. Entoloma luteum Peck (Hesler 1967) is a small species (10-25 mm broad pileus) characterized by an obtusely conical or at times subcampanulate, smoky golden-yellow pileus and stipe that are coated with innately brown fibrils or squamulose on the pileus disc over a golden or yellowish-green ground color, presence of large $(30-127 \times 7-12 \mu \mathrm{m})$ clavate cheilocystidia and lack of clamp connections.

The convex pileus, adnexed lamellae, long, twisted, silky striate stipe and cuboid spores indicates an affinity of E. flavoquadratum with subgenus Inocephalus.

\section{Acknowledgements}

Bijeesh $\mathrm{C}$ acknowledges financial support in the form of $\mathrm{PhD}$ fellowship (RGNF) from University Grants Commission, New Delhi.

\section{References}

Gates GM, Noordeloos ME. 2007 - Preliminary studies in the genus Entoloma in Tasmania-I. Persoonia 19, 157-226.

Hesler LR. 1967 -Entoloma in Southeastern North America. Beih. Nova Hedwigia 23, 1-196.

Horak E. 1976 - On cuboid species of Entoloma. Sydowia 28, 171-236.

Horak E. 1977 - Additions to "On cuboid-spored species of Entoloma". Sydowia 29, 289-299.

Kirk PM, Cannon PF, Minter DW, Stalpers JA. 2008 - Ainsworth \& Bisby's dictionary of the Fungi, $10^{\text {th }}$ ed. - CAB International, Wallingford.

Kornerup A, Wanscher JH. 1978 - Methuen Handbook of Colour. 3ed. Methuen, London.

Largent DL. 1994 - Entolomatoid fungi of the Western United States and Alaska. - Mad River Press, Eureka, California.

Largent DL, Johnson D, Watling R. 1977 - How to identify mushrooms to Genus III: Microscopic features - Mad River Press, Inc. California, 1-148.

Manimohan P, Joseph AV, Leelavathy KM. 1995 - The genus Entoloma in Kerala State, India. Mycological Research 99, 1083-1097.

Manimohan P, Leelavathy KM, Noordeloos ME. 2002 - Three new species of Entoloma from Kerala State, India. Persoonia 17, 625-630.

Manimohan P, Noordeloos ME, Dhanya AM. 2006 - Studies on the genus Entoloma (basidiomycetes, Agaricales) in Kerala State, India. Persoonia 19, 45-93.

Noordeloos ME, Hausknecht A. 2007 - The genus Entoloma (Basidiomycetes, Agaricales) of the Mascarenes and Seychelles. Fungal Diversity 27, 111-144.

Noordeloos ME, Morozova OV. 2010 - New and noteworthy Entoloma species from the Primorsky Territory, Russian Far East. Mycotaxon 112, 231-255. http://dx.doi.org/10.5248/112.231 
Noordeloos ME. 1981 - Introduction to the taxonomy of the genus Entoloma sensu lato (Agaricales). Persoonia 11, 121-151.

Noordeloos ME. 1992 - Entoloma s.l. Fungi Europaei, vol. 5. Giovanna Biella, Italy.

Noordeloos ME. 2004 - Entoloma s.l. Fungi Europaei, vol. 5a. Edizione Candusso, Italy.

Pegler DN. 1986 - Agaric flora of Sri Lanka. Kew Bulletin Additional Series 12, 1-519.

Pradeep CK, Shibu P Varghese, Vrinda KB, Baroni TJ. 2013 - Cuboid spored Entoloma in Kerala State, India. - Mycosphere 4, 333-344.

Pradeep CK, Vrinda KB, Shibu P Varghese, Baroni TJ. 2012 - New species of Entoloma (Basidiomycetes, Agaricales) from Kerala State, India. Mycotaxon120, 331-342. http://dx.doi.org/10.5248/120.331

Raj KNA, Latha KPD, Kumar TKA, Manimohan P 2015 - Rhodophana squamulosa-a new species of Entolomataceae from India. - Mycoscience 57, 90-95.

Raj KNA, Latha KPD, Kumar TKA, Manimohan P. 2014 - A new species of Entoloma from India. - Mycoscience 55, 400-404.

Raj KNA, Manimohan P. 2012 - A new species of Entoloma s.l. associated with earthworm casts. Mycosphere 3, 331-334, http://dx.doi.org/10.5943/mycosphere/3/3/6

Vellinga EC. 1988 - Glossary. In C Bas, Th W Kuyper, ME Noordeloos, Vellinga EC (eds.). Flora Agaricina Neerlandica 1, 54-64. A.A. Balkema, Rotterdam. 